\title{
A spectroscopic study of Be-like variable stars in the Small Magellanic Cloud
}

\author{
Beatriz Sabogal ${ }^{1}$, Alejandro García-Varela ${ }^{1}$ and \\ Ronald E. Mennickent ${ }^{2}$ \\ ${ }^{1}$ Departamento de Física, Universidad de los Andes, Bogotá, Colombia \\ email: bsabogal@uniandes.edu.co \\ ${ }^{2}$ Departamento de Astronomía, Facultad de Ciencias Físicas y Matemáticas, \\ Universidad de Concepción, Chile
}

\begin{abstract}
Photometric searches for Be stars in environments with different metallicities have led to the discovery of many Be-like star variables. The knowledge of these types of variables is still fragmentary. This work presents the preliminary results of analyzing FLAMES+GIRAFFE spectra of a sample of these Be-like stars that we have found in the Small Magellanic Cloud (SMC).
\end{abstract}

Keywords. stars: early-type, stars: emission-line, Be, stars: variables: other

\section{Introduction}

Mennickent et al. (2002) performed a photometric search for Be star candidates in the OGLE II database of SMC discovering new kind of variable stars in the same range of colours and magnitudes than classical Be stars. These objects were called Type-1 stars (showing outbursts), Type-2 stars (showing jumps), Type-3 stars (showing periodic or quasi-periodic variations) and Type- 4 stars (showing irregular variations). The same kind of variable stars were found in the Large Magellanic Cloud (Sabogal et al. 2005) and in the direction of the Galactic Bulge (Sabogal et al. 2008). Samples of Type-1 stars were studied by de Wit et al. (2006) and by Mennickent et al. (2009). These studies showed that Type-1 stars could be stars with an optically thick circumstellar envelope that evolves in an optically thin one before to dissipate into the interstellar medium. In order to follow up the study of these new variables, we are analyzing medium resolution spectra of 111 blue variable stars of the SMC in order to obtain clues to understand the mechanisms causing the different variations and their relationships with the Be phenomenon. The sample consists of 7 Type- 1 stars, 19 Type-2 stars, 43 Type-4, 18 Double Periodic Variables (DPVs) (Mennickent et al. 2003). The 24 remaining stars are periodic or multiperiodic Be stars, non-periodic Be stars, candidates to SPBs and non variable stars.

\section{Data}

A total of 3712 spectra were obtained in service mode with GIRAFFE/FLAMES spectrograph mounted at UT2/VLT/ESO telescope in Paranal Observatory (Chile). The LR MEDUSA mode was used, with a spectral range of $6437 \AA-7182 \AA$, and a central wavelength of $6822 \AA$. The resolving power was 8600 , and the average seeing was 0.8 arcsec. We have data for 5 epochs: October, November and December 2007, January and February 2008. The spectra were reduced and calibrated with GIRAFFE pipeline and normalized to the continuum using IRAF routine "continuum". 


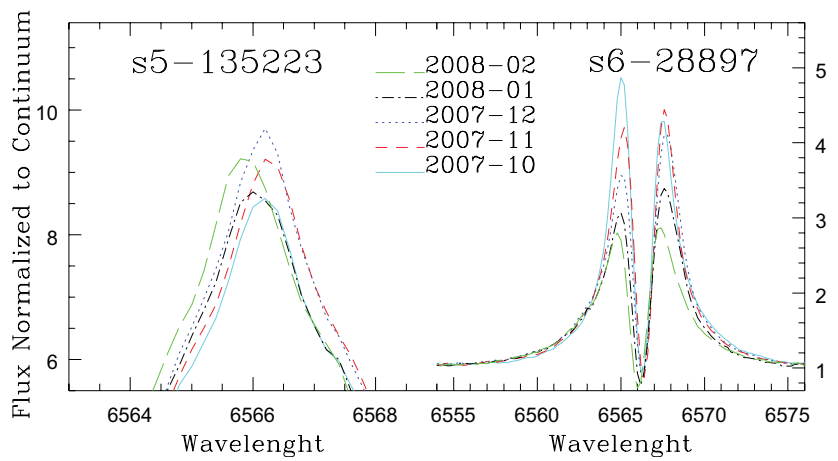

Figure 1. Variability of the $H_{\alpha}$ profile of a Type-1 star (Left: intensity increases during the first 3 months and proportionally decreases during the 2 following months) and a Type- 4 star (Right: intensity of the 2 components decreases during the five observation periods).

\section{Preliminary Results}

From visual inspection of the spectra of the total sample mentioned in previous section, it is evident that Type- 1 stars show strong and mostly double peaked $H_{\alpha}$ emission lines, although single and wine-bottle sharp profiles are also present (Fig. 1). Type-2 stars show double and single peaked $H_{\alpha}$ emission lines at the same proportion. The aspect of $H_{\alpha}$ lines of Type- 4 stars is similar to that of Type- 2 stars, although some of these Type- 4 stars have more complicated $H_{\alpha}$ profiles. DPVs also show both double and single peaked $H_{\alpha}$ emission lines. We found also one Type-4 star (smc-s5-196382) with an absorption $H_{\alpha}$ line. These preliminary results show that there is not a clear separation between the 4 classes of stars regarding their $H_{\alpha}$ profiles. Some of the double-peaked profiles show nearly equal $\mathrm{V}$ and $\mathrm{R}$ emission components but other stars have variable differences in the intensities of these emission components and only very slow variations in the equivalent width of the line (Fig. 1). It seems that a tendency between the time-scale of Type-1 star outbursts and the time-scale of changes in the strength of the $H_{\alpha}$ line exists, in agreement with the model of de Wit et al. (2006). This fact needs to be confirmed for all Type-1 stars with a follow up program obtaining simultaneosly photometric and spectroscopic data for such stars. It is also possible that a correspondence exists between the constant state of Type- 2 stars during a long time-scale and the small changes in the intensity of their $H_{\alpha}$ lines. This needs to be confirmed too.

Double-peaked $H_{\alpha}$ profiles resemble those of Be stars and indicate the presence of a flattened, equatorial Keplerian disk that is seen by an observer viewing the star at an intermediate or edge-on angle. The $H_{\alpha}$ profiles shown in Fig. 1 (right panel), presenting changes in the intensities of the blue and red emission components, are commonly explained by the appearance and slow drift of a disc-like region of enhanced gas density. These aspects provide important evidence that the 4 classes of stars studied actually are Be stars and that their variability is associated with the Be phenomenon.

\section{References}

de Wit, W. J., Lamers, H. J. G. L. M., Marquette, J. B., \& Beaulieu, J. P. 2006, A\&SA, 456, 1027

Mennickent, R. E., Pietrzyński, G., Gieren, W., \& Szewczyk, O. 2002, A\&\&A, 393, 887

Mennickent, R. E., Pietrzyński, G., Diaz, M., \& Gieren, W. 2003, A\&\&A, 399, L47

Mennickent, R. E., Sabogal, B., Granada, A., \& Cidale, L. 2009, PASP, 121, 125

Sabogal, B. E., Mennickent, R. E., Pietrzyński, G., \& Gieren, W. 2005, MNRAS, 361, 1055

Sabogal, B. E., Mennickent, R. E., Pietrzyński, G., García, J. A. et al. 2008, A\& $A, 478,659$ 